\title{
Attitude and associated factors of COVID-19 vaccine acceptance among health professionals in Debre Tabor Comprehensive Specialized Hospital, North Central Ethiopia; 2021: cross- sectional study
}

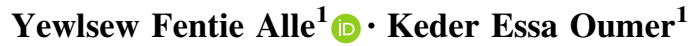

Received: 14 April 2021 / Accepted: 8 June 2021 / Published online: 25 June 2021

(C) Indian Virological Society 2021

\begin{abstract}
Control of coronavirus disease through vaccination is not merely dependent on vaccine efficacy and safety. Professional and general public acceptance is vital for the successful control of the virus. This study aimed (1) to assess health professionals' attitude towards COVID-19 vaccine acceptance; (2) to identify factors associated with health professional's attitude towards COVID-19 vaccine acceptance. An Institutional based cross-sectional study was conducted on health professionals working at Debre Tabor Comprehensive Specialized Hospital. A structured questionnaire containing 16 items was used to assess the attitude of health professionals on vaccine acceptance. Bivariable and multivariable logistic analysis was done to identify factors associated with the attitude of health professionals. In this study, 42.3\% [95\% CI (36.7-47.6)] participants had positive attitude to COVID-19 vaccine acceptance. Age 30-39 years [AOR 2.23; (CI 1.23-4.04)] and $\geq 40$ years [AOR 5.51; (CI 2.47-12.30)] of respondents have positive attitude than their counterparts. Also physicians [AOR 3.67; (CI 1.90-7.09)], pharmacists [AOR 4.27; (CI 1.39-13.09)] and laboratory professionals [AOR 4.56; (CI 1.34-15.39)] have higher attitude to COVID vaccine acceptance than nurses. In conclusion, the attitude level of health professionals on COVID-19 vaccine acceptance was poor. Age and profession were factors significantly associated with the attitude level of health professionals for COVID-19 vaccine acceptance.
\end{abstract}

Yewlsew Fentie Alle

fyewlsew@gmail.com

1 Department of Anesthesia, College of Health Sciences, School of Medicine, Debre Tabor University, P.O. Box: 272, Debre Tabor, Ethiopia
Keywords Health professionals · Attitude - Vaccine . Acceptance

$\begin{array}{ll}\text { Abbreviations } \\ \text { AOR } & \text { Adjusted Odds Ratio } \\ \text { CDC } & \text { Communicable Disease Control } \\ \text { COR } & \text { Crude Odds Ratio } \\ \text { COVID-19 } & \text { Corona Virus Disease-2019 } \\ \text { SNNP } & \text { South Nation Nationalities and Peoples } \\ \text { SPSS } & \text { Statistical Package for Social Science } \\ \text { USA } & \text { United States of America } \\ \text { WHO } & \text { World Health Organization }\end{array}$

\section{Introduction}

Vaccines are life-saving inventions that have been responsible for the elimination and control of many infectious diseases in many parts of the world $[14,11]$. In addition to providing direct immunity and preventing disease among vaccinated individuals, they have been shown to protect unvaccinated individuals through herd immunity, if a greater proportion of the population is immune $[14,13]$. Since the occurrence of pandemic COVID 19, WHO and Health care institutions are working on prevention, diagnosis, and treatment including the development of vaccines [10].

Control of coronavirus disease (COVID-19) through vaccination is not merely dependent on vaccine efficacy and safety. Instead, the vaccine needs also to have health professionals and general public acceptance for successful control of the virus [4-24]. 
Healthcare workers are being prioritized among the high-risk groups who will be considered as initial candidates for early vaccination. However, there is so much research studying factors that influenced the acceptance of vaccinations among the general population and health professionals of which included political party orientation, doubts toward expedited development/approval process, and perceived political interference. Many receptive participants preferred to wait until others have taken the vaccine; mandates that could increase resistance are unique for COVID [20]. Like the previous vaccines, the COVID19 vaccine will be a big challenge to address [28]. In a recent survey, more than one-third of lay respondents were unsure or did not intend to take the vaccine for different reasons and beliefs $[14,28,18]$.

Health care workers are a reliable source of information for vaccines and Health information delivered by them can improve adherence to vaccination recommendations. Thus, the role of healthcare workers becomes particularly important in advising patients and communities, and as well as through role modeling behavior. Nevertheless, health professionals are hesitant on COVID 19 vaccination most of them have an issue with the safety they thought it developed within a short period $[10,8]$. So it is important to consider healthcare workers' attitudes about COVID-19 vaccination to overcome barriers and to have effective coverage of vaccination.

Therefore the objective of this study is to determine the attitude and associated factors towards COVID 19 Vaccine acceptance among healthcare workers working in Debre Tabor Comprehensive Specialized Hospital.

\section{Methods}

\section{Study area and period}

The study was conducted in Ethiopia at the South Gondar zone of Debre Tabor Comprehensive Specialized Hospital from February 5 to March 20, 2021. Debre Tabor Comprehensive Specialized Hospital is found in Debre Tabor Town and South Gondar Zone is among one of the 10 administrative zones in the Amhara region. The Town is found about $669 \mathrm{~km}$ northwest of Addis Ababa, the capital city of Ethiopia, and $97 \mathrm{~km}$ southwest of Bahir Dar, the capital city of the Amhara region. This town has a latitude and longitude of $11^{\circ} 51^{\prime} \mathrm{N} 38^{\circ} 1^{\prime} \mathrm{E} / 11.850^{\circ} \mathrm{N} 38.017^{\circ} \mathrm{E}$ with an elevation of $2706 \mathrm{~m}$ above sea level. The Hospital is the only Comprehensive Specialized Hospital in the South Gondar Zone.

\section{Study design}

An Institutional based cross-sectional survey was conducted on health professionals working in South Gondar zone Debre Tabor Comprehensive Specialized Hospital.

\section{Study populations}

All health professionals working at Debre Tabor Comprehensive Specialized Referral Hospitals of North Central Ethiopia were the study populations.

\section{Source populations}

All physicians, Anesthetists, Nurses, Midwives, Pharmacists, and laboratory professionals that work at Debre Tabor Comprehensive Specialized Referral Hospitals of North Central Ethiopia during the study period were source populations.

\section{Inclusion criteria}

All physicians, Anesthetists, Nurses, Midwives, Pharmacists, and laboratory professionals that work at Debre Tabor Comprehensive Specialized Referral Hospitals of North Central Ethiopia during the study period.

\section{Exclusion criteria}

Health professionals with sick, annual, and maternity leave during the study period and those unwilling to participate in the study were excluded.

\section{Dependent variable}

Attitude level of health professionals towards COVID-19 vaccine acceptance.

\section{Independent variable}

Socio-demographic characteristics gender, age, marital status, religion, ethnicity, educational level, year of experience, presence of the family member, and monthly income.

\section{Sample size determination and sampling procedure}

The predetermination of sample size was not done but, an institutional-based survey that included all health professionals working in the hospital during the data collection period was studied. 


\section{Data collection instrument and procedures}

The attitude level of health professionals measured with a data collection tool adapted from a study done in the USA on health care workers [28]. The data collection instrument has 16 attitude questions measured with a five-point Likert scale (strongly disagree, disagree, neutral, agree, and strongly agree). Before the application of this tool in our setup, it was evaluated by the expert committee of Debre Tabor University and got approval on applicability in our setup without modification. The data was collected using a structured self-administered questionnaire which was distributed to the study participants by data collectors. Six health professionals' in which one data collector from each department (physician, anesthetist, pharmacist, laboratory professional, nurse, and midwife) collected the data after they were given adequate training.

\section{Data quality assurance}

To assure the data collection instrument pretest was done on $5 \%$ of study participants that work at Debre Markos referral hospital. One day of training was given for data collectors and the collected data was checked for its completeness by all data collectors.

\section{Data entry and analysis}

The data were entered into Epidata version 4.2.0.0 and exported to SPSS version 20 for analysis.

Descriptive statistics were carried out, and both bivariable and multivariable logistic regression analyses were used to identify factors associated with the attitude level of health professionals to COVID-19 vaccine acceptance. Variables with a $p$-value of less than $<0.2$ in the bivariable logistic analysis were fitted into a multivariable logistic regression analysis and a multicollinearity test was done. Both crude odds ratio (COR) in bivariable logistic regression and adjusted odds ratio (AOR) in multivariable logistic regression with the corresponding $95 \%$ confidence interval were calculated to show the strength of association. In multivariable logistic regression analysis, variables with a $p$-value $<0.05$ were considered statistically significant.

\section{Ethical clearance}

Ethical clearance was obtained from Debre Tabor University Ethical Review Committee of College of Health Science Research and Community Service Coordination Service. A permission letter was obtained from Debre Tabor Comprehensive Specialized Hospital and informed consent was taken from all study participants after telling the objective and significance of the study. The confidentiality and anonymity of study participants' responses were ensured.

\section{Operational definitions}

Positive attitude: when study participants respond 50\% or more of attitude questions.

Negative attitude: when study participants respond to less than $50 \%$ of attitude questions.

\section{Result}

\section{Socio-demographic characteristics of study participants}

A total of 327 study participants were involved, but about 319 participants completely respond to the survey with a 97.6\% response rate. Eight incomplete questioners are excluded from the analysis. The majority of study participants were males $203(63.6 \%)$ and 157 (49.2\%) participants were between the age of 30-39 years. About 260 $(81.5 \%)$ were married and $269(84.3 \%)$ have family members. The dominant ethnic group and religion of study participants were Amhara 260 (81.5\%) and Orthodox 225 (70.5\%). A higher number of study participants were nurses $128(40.1 \%)$. Of study participants, $53.3 \%$ of them were BSc and below and $50.5 \%$ were with work experience of $1-5$ years. About $42.6 \%$ of study participants have greater than 9000 Ethiopian birr monthly income (Table 1).

\section{Attitude level of health professionals for COVID -19 vaccine acceptance}

About 135 (42.3\%) [95\% CI (36.7-47.6)] study participants had positive attitude to COVID-19 vaccine acceptance (Fig. 1). About 54 (62.8\%) physicians, 11 (64.7\%) Pharmacists, 10 (66.7\%) laboratory professionals, 9 (36.0\%) Anesthetists, 39 (30.5\%) Nurses and 12 (25.0\%) midwives had positive attitude of COVID-19 vaccination.

In this study, the Median attitude score of respondents towards COVID-19 vaccination was 38 (IQR 23, 31-54, Range 18-80). About 129 (40.4\%) of study participants agreed on they don't trust the information provided by the government about COVID severity and $28(8.8 \%)$ disagreed. Also, only $30(9.4 \%)$ of study participants agreed that they don't trust doctors recommending Vaccines while 85 (26.6\%) disagreed they don't trust doctor's recommendation of COVID vaccination (Table 2). 
Table 1 Socio-demographic characteristics of health professionals $(\mathrm{n}=319)$

\begin{tabular}{|c|c|c|}
\hline Variables & Frequency (319) & Percentage $(100 \%)$ \\
\hline \multicolumn{3}{|l|}{ Age (years) } \\
\hline $20-29$ & 113 & 35.4 \\
\hline $30-39$ & 157 & 49.2 \\
\hline$\geq 40$ & 49 & 15.4 \\
\hline \multicolumn{3}{|l|}{ Sex } \\
\hline Male & 203 & 63.6 \\
\hline Female & 116 & 36.4 \\
\hline \multicolumn{3}{|l|}{ Profession } \\
\hline Physician & 86 & 27.0 \\
\hline Anesthetist & 25 & 7.8 \\
\hline Nurse & 128 & 40.1 \\
\hline Midwife & 48 & 15.0 \\
\hline Pharmacy & 17 & 5.3 \\
\hline Laboratory & 15 & 4.7 \\
\hline \multicolumn{3}{|l|}{ Marital status } \\
\hline Unmarried & 59 & 18.5 \\
\hline Married & 260 & 81.5 \\
\hline \multicolumn{3}{|l|}{ Having family members } \\
\hline Yes & 269 & 84.3 \\
\hline No & 50 & 15.7 \\
\hline \multicolumn{3}{|l|}{ Religion } \\
\hline Orthodox Christian & 225 & 70.5 \\
\hline Protestant & 65 & 20.4 \\
\hline Muslim & 23 & 7.2 \\
\hline Others & 6 & 1.9 \\
\hline \multicolumn{3}{|l|}{ Ethnicity } \\
\hline Amhara & 260 & 81.5 \\
\hline Oromo & 12 & 3.8 \\
\hline Tigrie & 20 & 6.3 \\
\hline SNNP & 23 & 7.2 \\
\hline Others & 4 & 1.3 \\
\hline \multicolumn{3}{|l|}{ Educational level } \\
\hline BSc degree and below & 170 & 53.3 \\
\hline MSc and above & 149 & 46.7 \\
\hline \multicolumn{3}{|l|}{ Work experience (years) } \\
\hline $1-5$ & 161 & 50.5 \\
\hline $6-10$ & 116 & 36.4 \\
\hline$>10$ & 42 & 13.2 \\
\hline \multicolumn{3}{|l|}{ Income (Ethiopian birr) } \\
\hline$<3000$ & 16 & 5.0 \\
\hline $3000-6000$ & 77 & 24.1 \\
\hline $6001-9000$ & 90 & 28.2 \\
\hline$>9000$ & 136 & 42.6 \\
\hline
\end{tabular}

Factors Associated health professionals attitude regarding COVID-19 vaccine acceptance

In the bivariable logistic analysis, the attitude of health professionals on COVID-19 vaccine acceptance was significantly associated with age, profession, religion, and ethnicity. The multivariable logistic analysis showed that health professionals with the age of 40 or above 5.51 (AOR $5.51 ; 95 \%$ CI $2.47-12.30$ ) and age with $30-39$ years 2.23 (AOR 2.23; 95\% CI 1.23-4.04) were more to have a positive attitude to COVID-19 vaccination than professionals within the age of 20-29 years. The odds of physicians being positive attitude was 3.67 (AOR 3.67; 95\% CI 1.90-7.09) than nurses. Pharmacists were 4.27 (AOR 4.27; 95\% CI 1.39-13.09) times more positive for COVID vaccine acceptance than nurses while laboratory professionals were 4.56 (AOR 4.56; 95\% CI 1.34-15.39) time more positive towards vaccine acceptance (Table 3 ).

\section{Discussion}

COVID-19 spread is dramatically increasing in Africa and based on daily reports of the ministry of health in Ethiopia, the spread is a devastating and great concern for the country [11-13]. COVID-19 with multiple challenges is greatly affecting all systems of the country of Ethiopia and it is alarming for the gov't, stakeholders, and every citizen of the country to combat the pandemic [171]. In Ethiopia, overall health professional's knowledge, attitude, and practice to COVID-19 are poor [19]. Also, low-level preparedness to prevent the pandemic with limited personal protective equipment supplies contributes to the rapid spread of COVID-19 in Ethiopia [17-19]. The preparedness level to combat the pandemic in the study area was low and daily reports of COVID-19 positive patients are rapidly increasing [29].

Currently, WHO, CDC, and other collaborative organizations plan to implement the COVID-19 vaccine globally with optimal safety and minimal cost [21-23]. Health professionals are front lines to be affected by the pandemic and should be exemplary to take the vaccine and promote the importance of the vaccine for effective prevention to the community [154]. Ethiopia currently starts vaccination primarily on health professionals, high risks, and communities that live in major cities [6]. Health professionals' intention for vaccination is not yet known but, community acceptance for vaccination was low [6].

In the current study, the attitude of health professionals for COVID vaccination was low, about $42.3 \%$ this result was higher than studies done in the Democratic Republic of Congo $27.7 \%$ and USA $36 \%$ [28, 23] this variation may be the time frame that data was taken before the vaccine was 
Fig. 1 Attitude level of health professionals for COVID-19 vaccine acceptance
Attitude of health professionals to COVID-19 vaccine acceptance

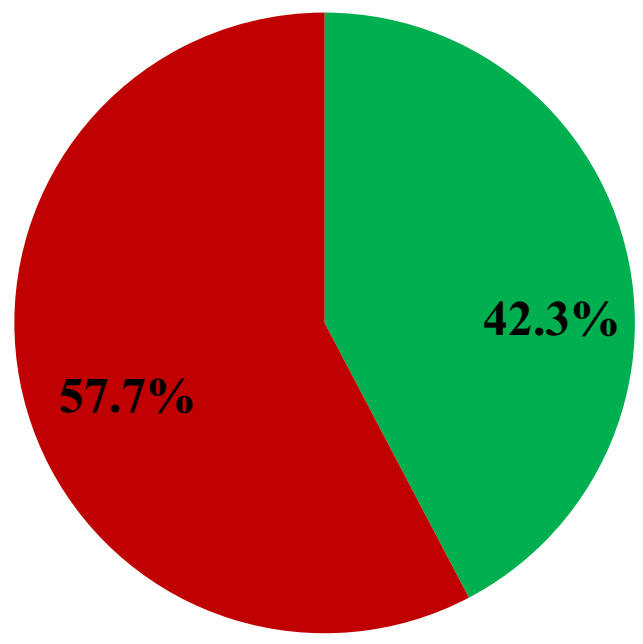

- Possitive attitude

Negative attitude

Table 2 Attitude of health professionals regarding COVID-19 acceptance ( $\mathrm{n}=319)$

\begin{tabular}{|c|c|c|c|c|c|}
\hline Attitude towards COVID-19 vaccine acceptance & $\mathrm{SD}(\%)$ & DA $(\%)$ & $\mathrm{N}(\%)$ & $\mathrm{A}(\%)$ & $\mathrm{SA}(\%)$ \\
\hline I do not believe vaccine works & $27(8.5)$ & $57(17.9)$ & $57(17.9)$ & $98(30.7)$ & $80(25.1)$ \\
\hline I don't believe vaccines are safe & $40(12.5)$ & $42(13.2)$ & $39(12.2)$ & $115(36.1)$ & $83(26.0)$ \\
\hline I don't get vaccinated for religious reasons & $116(36.4)$ & $73(22.9)$ & $47(14.7)$ & $38(11.9)$ & $44(13.8)$ \\
\hline I don't get vaccinated for personal freedom of choice & $35(11.0)$ & $58(18.2)$ & $42(13.2)$ & $97(30.4)$ & $87(27.3)$ \\
\hline I do not get vaccinated because of fear of needles & $72(22.6)$ & $69(21.6)$ & $40(12.5)$ & $74(23.2)$ & $64(20.1)$ \\
\hline I am worried about Sid effects of a covd-19 vaccine & $25(7.8)$ & $60(18.8)$ & $49(15.4)$ & $93(29.2)$ & $92(28.8)$ \\
\hline I am worried about effectiveness of COVID vaccine & $51(16.0)$ & $51(16.0)$ & $44(13.8)$ & $95(29.8)$ & $94(29.5)$ \\
\hline I am worried about the pocket cost of the vaccine & $41(12.9)$ & $72(22.6)$ & $44(13.8)$ & $94(29.5)$ & $68(21.3)$ \\
\hline I am worried about the adverse effects of the vaccine on preexisting conditions & $30(9.4)$ & $48(15.0)$ & $39(12.2)$ & $97(30.4)$ & $104(32.6)$ \\
\hline I do not need a vaccine for my risk level & $31(9.8)$ & $58(18.2)$ & $46(14.4)$ & $97(30.4)$ & $86(27.0)$ \\
\hline $\begin{array}{l}\text { I have a prior reaction to other vaccines and I am worried about the reaction to } \\
\text { COVID vaccination }\end{array}$ & $97(30.4)$ & $104(32.6)$ & $39(12.2)$ & $38(11.9)$ & $41(12.9)$ \\
\hline I am worried about the rapidity of the development of the COVID vaccine & $32(10.0)$ & $36(11.3)$ & $45(14.1)$ & $117(36.7)$ & $89(27.9)$ \\
\hline I don't trust pharmaceutical companies making vaccine & $33(10.3)$ & $29(9.1)$ & $38(11.9)$ & $105(32.9)$ & $114(35.7)$ \\
\hline I don't trust FDA/CDC overseeing the Vaccine development & $31(9.7)$ & $31(9.7)$ & $28(8.8)$ & $117(36.7)$ & $112(35.1)$ \\
\hline I don't trust doctors recommending Vaccine & $137(42.9)$ & $85(26.6)$ & $45(14.1)$ & $30(9.4)$ & $31(9.7)$ \\
\hline I don't trust the information provided by the government about COVID severity & $41(12.9)$ & $28(8.8)$ & $33(10.3)$ & $129(40.4)$ & $88(27.6)$ \\
\hline
\end{tabular}

$S D$ strongly disagree, $D A$ disagree, $N$ neutral, $A$ agree, $S A$ strongly agree

given to the major of world. But another study done in the USA $57.5 \%$ and Greek $78.5 \%$ showed that health professionals have great intentions to be vaccinated $[27,25]$. The possible explanation for this might be variation in knowledge level about the vaccine, variation in governmental policies, and coverage of social media.

This study showed that age was associated with an attitude of COVID vaccine acceptance and respondents with age 30-39 with AOR 2.23 (1.23-4.04) and 40 or above AOR 5.51 (2.47-12.30) have positive attitude compared to their counterparts. This result nearly similar to a study done in Greek in which age greater than 45 years were OR 2.01 (1.36-12.3) associated with positive attitude [25]. The possible reason for this might be as age increases the chance of comorbid illness increases and cause a high risk for COVID -19, so this increases intension for vaccination [27].

In this study, the profession was another factor associated with an attitude of health professionals to COVID-19 vaccine acceptance and physicians were AOR 3.67 (1.90-7.09) times more positive for vaccine acceptance. This result is supported by a study done in the Democratic 
Table 3 Factors associated with health professionals towards COVID-19 vaccine acceptance

\begin{tabular}{|c|c|c|c|c|c|}
\hline \multirow[t]{2}{*}{ Variables } & \multicolumn{2}{|l|}{ Attitude level } & \multirow{2}{*}{$\begin{array}{l}\text { Crude odds ratio } \\
95 \% \text { CI }\end{array}$} & \multirow{2}{*}{$\begin{array}{l}\text { Adjusted odds ratio } \\
95 \% \mathrm{CI}\end{array}$} & \multirow[t]{2}{*}{$p$-value } \\
\hline & $\begin{array}{l}\text { Positive } \\
135(42.3 \%)\end{array}$ & $\begin{array}{l}\text { Negative } \\
184(57.7 \%)\end{array}$ & & & \\
\hline \multicolumn{6}{|l|}{ Age (years) } \\
\hline $20-29$ & $29(25.7 \%)$ & $84(74.3 \%)$ & 1 & & \\
\hline $30-39$ & $72(45.9 \%)$ & $85(54.1 \%)$ & $2.63(1.03,6.69)$ & $2.23(1.23,4.04)$ & $0.008 *$ \\
\hline$\geq 40$ & $34(69.4 \%)$ & $15(30.6 \%)$ & $6.11(1.39,26.92)$ & $5.51(2.47,12.30)$ & $0.000 *$ \\
\hline \multicolumn{6}{|l|}{ Profession } \\
\hline Physician & $54(62.8 \%)$ & $32(37.2 \%)$ & $2.80(1.09,7.24)$ & $3.67(1.90,7.09)$ & $0.000 *$ \\
\hline Anesthetist & $9(36.0 \%)$ & $16(64.0 \%)$ & $0.79(0.26,2.37)$ & $1.14(0.43,2.99)$ & 0.791 \\
\hline Nurse & $39(30.5 \%)$ & $89(69.5 \%)$ & 1 & & \\
\hline Midwife & $12(25.0 \%)$ & $36(75.0 \%)$ & $0.57(0.24,1.34)$ & $0.72(0.32,1.61)$ & 0.419 \\
\hline Pharmacy & $11(64.7 \%)$ & $6(35.3 \%)$ & $3.82(1.13,12.97)$ & $4.27(1.39,13.09)$ & $0.011 *$ \\
\hline Laboratory & $10(66.7 \%)$ & $5(33.3 \%)$ & $4.56(1.26,16.48)$ & $4.56(1.34,15.39)$ & $0.015^{*}$ \\
\hline \multicolumn{6}{|l|}{ Religion } \\
\hline Orthodox & $105(46.7 \%)$ & $120(53.3 \%)$ & 1 & & \\
\hline Protestant & $18(27.7 \%)$ & $47(72.3 \%)$ & $0.57(0.26,1.26)$ & $0.49(0.23,1.04)$ & 0.063 \\
\hline Muslim & $9(39.1 \%)$ & $14(60.9 \%)$ & $0.57(0.18,1.78)$ & $0.61(0.20,1.84)$ & 0.382 \\
\hline Others & $3(50.0 \%)$ & $3(50.0 \%)$ & $1.85(0.23,14.69)$ & $1.55(0.23,10.47)$ & 0.654 \\
\hline \multicolumn{6}{|l|}{ Ethnicity } \\
\hline Amhara & $115(44.2 \%)$ & $145(55.8 \%)$ & 1 & & \\
\hline Oromo & $5(41.7 \%)$ & $7(58.3 \%)$ & $0.63(0.14,2.80)$ & $1.06(0.27,4.12)$ & 0.939 \\
\hline Tigrie & $7(35.0 \%)$ & $13(65.0 \%)$ & $2.69(0.81,8.92)$ & $1.93(0.62,5.97)$ & 0.254 \\
\hline SNNP & $7(30.4 \%)$ & $16(69.6 \%)$ & $0.45(0.12,1.62)$ & $0.44(0.13,1.56)$ & 0.191 \\
\hline Others & $1(25.0 \%)$ & $3(75.0 \%)$ & $0.80(0.06,10.21)$ & $0.69(0.06,8.27)$ & 0.768 \\
\hline
\end{tabular}

$*_{p}$ value $<0.05,1=$ reference

Republic of Congo which showed that physicians' OR 1.59 (1.02-2.44) time to have a positive attitude [23]. The reason for this might be variation in the educational background may affect attitude. Also being pharmacist AOR 4.27 (1.39-13.09) and laboratory professional AOR 4.56 (1.34-15.39) were more likely to have a positive attitude than their counterparts. The possible explanation for this might professional related clinical exposure to COVID-19 might increase the perception of the vaccine's importance and attitude.

This study shows a poor attitudinal level of health professionals for COVID-19 vaccination, so the government and policymakers in the health sectors of Ethiopia should address the concerns of health professionals and give continuous professional development training on the effectiveness and safety of COVID-19 vaccines to build an attitude of health professionals on vaccine acceptance.

\section{Limitations of the study}

One limitation of the present study was that single health institution professionals were studied which might not generalize other organizations' health professionals' attitude levels. Additionally, a comparison of attitudinal level with health professionals and community was not done. Furthermore, additional studies are required to establish whether the results of the present study are similar to those found in other health institution professionals.

\section{Conclusion}

The attitude level of health professionals on COVID-19 vaccine acceptance was less than $50 \%$ which is indicative of a poor attitude to accept vaccination. Age and profession were factors significantly associated with the attitude level of health professionals for COVID-19 vaccine acceptance. So, since health professionals are front liners to combat the pandemic, mandatory measures are required to achieve proper vaccine acceptance and coverage.

Acknowledgements We would like to acknowledge Debre Tabor University for ethical permission and Debre Tabor Comprehensive Specialized Hospital for giving permission letter also; all study participants for their co-operation during data collection. 
Funding Self-funded.

\section{Declarations}

Conflict of interest There is no conflict of interest.

Ethical approval and consent to participate To keep the ethical soundness of the research, an ethical approval letter was obtained from Debre Tabor University. Written consent was also secured before data collection.

Data sharing statement The data will be shared upon reasonable request.

\section{References}

1. Ayenew B, Pandey D. Challenges and opportunities to tackle COVID-19 spread in Ethiopia. J PeerSci. 2020;2(2):e1000014.

2. Bell BP, Romero JR, Lee GM. Scientific and ethical principles underlying recommendations from the Advisory Committee on Immunization Practices for COVID-19 vaccination implementation. JAMA. 2020;324(20):2025-6.

3. Biadgilign S, Yigzaw M. COVID-19 in Ethiopia: current situation, missed opportunities, and the risk of health system disruptions. Pan Afr Med J. 2020;35(Suppl 2):66.

4. Chersich MF, et al. COVID-19 in Africa: care and protection for frontline healthcare workers. Glob Health. 2020;16:1-6.

5. DeRoo SS, Pudalov NJ, Fu LY. Planning for a COVID-19 vaccination program. JAMA. 2020;323(24):2458-9.

6. Dereje N, et al. COVID-19 vaccine hesitancy in Addis Ababa, Ethiopia: a mixed-methods study. medRxiv. 2021.

7. Deressa W, et al. Availability of personal protective equipment and satisfaction of healthcare professionals during COVID-19 pandemic in Ethiopia. medRxiv. 2020.

8. Di Martino G, et al. Knowledge and attitude towards vaccination among healthcare workers: a multicenter cross-sectional study in a southern Italian region. Vaccines. 2020;8(2):248.

9. Dooling K. The Advisory Committee on Immunization Practices' updated interim recommendation for allocation of COVID-19 vaccine-United States, December 2020. Morb Mortal Wkly Rep: MMWR. 2021;69.

10. Dror AA, et al. Vaccine hesitancy: the next challenge in the fight against COVID-19. Eur J Epidemiol. 2020;35(8):775-9.

11. Dubé E. Addressing vaccine hesitancy: the crucial role of healthcare providers. Clin Microbiol Infect. 2017;23(5):279-80.

12. Emeto TI, Alele FO, Ilesanmi OS. Evaluation of the effect of border closure on COVID-19 incidence rates across nine African countries: an interrupted time series study. Trans R Soc Trop Med Hyg. 2021. https://doi.org/10.1093/trstmh/trab033.

13. Fine P, Eames K, Heymann DL. "Herd immunity": a rough guide. Clin Infect Dis. 2011;52(7):911-6.
14. Fisher KA, et al. Attitudes toward a potential SARS-CoV-2 vaccine: a survey of US adults. Ann Intern Med. 2020;173(12):964-73.

15. French J, et al. Key guidelines in developing a pre-emptive COVID-19 vaccination uptake promotion strategy. Int J Environ Res Public Health. 2020;17(16):5893.

16. Getaneh Y, et al. Global lessons and potential strategies in combating COVID-19 pandemic in Ethiopia: systematic review. medRxiv. 2020.

17. Goshu D, et al. Economic and welfare effects of COVID-19 and responses in Ethiopia: initial insights. 2020.

18. Khan YH, et al. Threat of COVID-19 vaccine hesitancy in Pakistan: the need for measures to neutralize misleading narratives. Am J Trop Med Hyg. 2020;103(2):603-4.

19. Lake EA, et al. Knowledge, attitude and practice towards COVID-19 among health professionals in Ethiopia: a systematic review and meta-analysis. PLoS ONE. 2021;16(2):0247204.

20. Lin C, Tu P, Beitsch LM. Confidence and receptivity for COVID19 vaccines: a rapid systematic review. Vaccines. 2021;9(1):16.

21. Mulu GB, et al. Preparedness and responses of healthcare providers to combat the spread of COVID-19 among North Shewa Zone Hospitals, Amhara, Ethiopia, 2020. Infect Drug Resist. 2020;13:3171.

22. Nkengasong JN, Mankoula W. Looming threat of COVID-19 infection in Africa: act collectively, and fast. Lancet. 2020;395(10227):841-2.

23. Nzaji MK, et al. Acceptability of vaccination against COVID-19 among healthcare workers in the Democratic Republic of the Congo. Pragmat Obs Res. 2020;11:103.

24. Omer SB, et al. Vaccine refusal, mandatory immunization, and the risks of vaccine-preventable diseases. $\mathrm{N}$ Engl $\mathrm{J}$ Med. 2009;360(19):1981-8.

25. Papagiannis D, et al. Acceptability of COVID-19 vaccination among Greek health professionals. Vaccines. 2021;9(3):200.

26. Sallam M. COVID-19 vaccine hesitancy worldwide: a concise systematic review of vaccine acceptance rates. Vaccines. 2021;9(2):160.

27. Shaw J, et al. Assessment of US health care personnel (HCP) attitudes towards COVID-19 vaccination in a large university health care system. Clin Infect Dis. 2021. https://doi.org/10.1093/ $\mathrm{cid} / \mathrm{ciab} 054$.

28. Shekhar R, et al. COVID-19 vaccine acceptance among health care workers in the United States. Vaccines. 2021;9(2):119.

29. Tiruneh A, et al. A cross-sectional survey of COVID-19 preparedness in governmental hospitals of North-West Ethiopia. SAGE Open Med. 2021;9:2050312121993292.

Publisher's Note Springer Nature remains neutral with regard to jurisdictional claims in published maps and institutional affiliations. 